\title{
COVID-19-Risiko bei Demenzkranken verdoppelt
}

COVID-19 wird bei Demenzkranken rund doppelt so oft diagnostiziert wie bei älteren pflegebedürftigen Menschen ohne Demenz. Patienten mit vaskulärer Demenz sind besonders häufig betroffen.

\begin{abstract}
Demenzkranke sind besonders COVID-19-gefährdet - das ist zunächst wenig überraschend, da sie häufig in Pflegeeinrichtungen leben, in denen es immer wieder zu gravierenden SARS-CoV-2-Ausbrüchen kommt. Dies sei aber nicht der einzige Grund, berichten Experten um Dr. Quan Qiu Wang von der Case Western Reserve University in Cleveland, USA: Die Gefahr, an COVID-19 zu erkranken, ist ihren Erkenntnissen zufolge bei Demenzkranken unabhängig vom Alter und der Betreuungssituation rund doppelt so hoch wie bei älteren Menschen ohne Demenz [1].

Die Forscher um Wang haben für ihre Analyse im August 2020 elektronische Krankenakten von 62 Millionen erwachsenen US-Amerikanern nach COVID-19- und Demenzdia-

gnosen durchforstet. In über einer Million Akten war eine Demenzdiagnose vermerkt, knapp 16.000-mal eine COVID-19-Diagnose und 810-mal beides. Anfang August hatten sich in den USA offiziellen Angaben zufolge aber schon fünf Millionen Bürger mit SARS-CoV-2 infiziert, danach deckten die elektronischen Krankenakten zu diesem Zeitpunkt nur einen winzigen Bruchteil der tatsächlich Erkrankten ab.

Berücksichtigten die Forscher nur demografische Faktoren, also Alter, Geschlecht und Ethnie, so kamen sie auf ein dreifach erhöhtes COVID-19-Risiko unter Demenzkranken. Adjustierten sie zusätzlich für Begleiterkrankungen und bekannte COVID-19-Risikofaktoren, darunter auch der Aufenthalt in einer Pflegeeinrichtung, so fanden sie immer noch ein
\end{abstract}

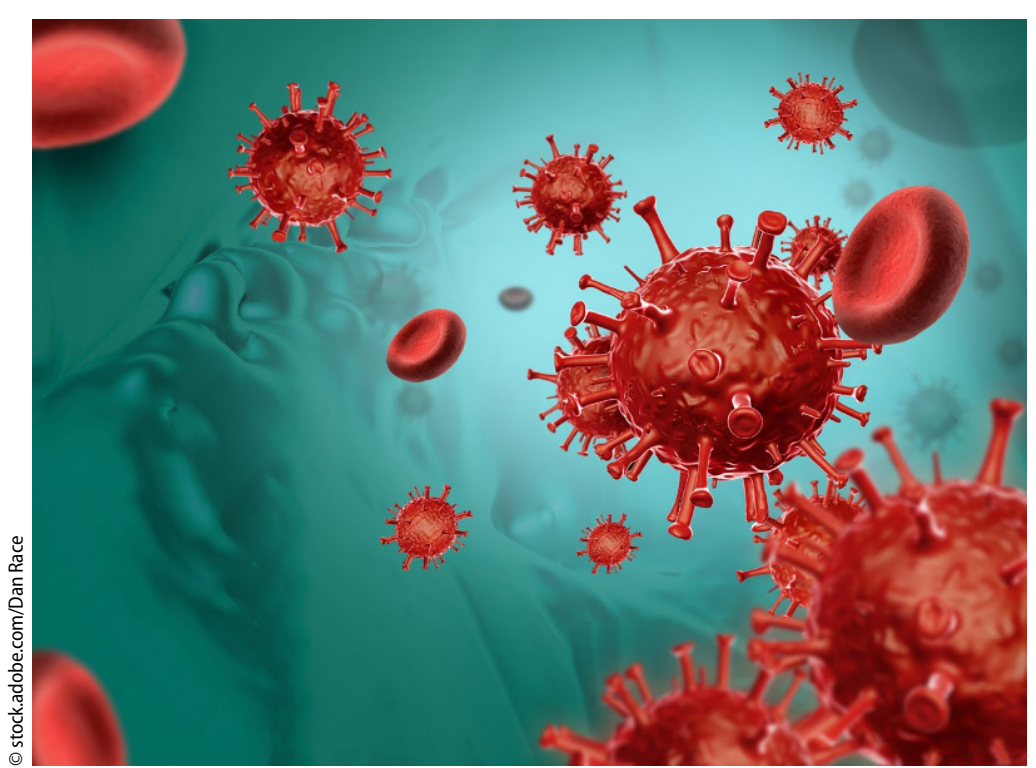

$\Delta$ COVID-19 wird bei Demenzkranken rund doppelt so oft diagnostiziert wie bei älteren pflegebedürftigen Menschen ohne Demenz verdoppeltes COVID-19-Risiko. Danach wäre das COVID-19-Risiko für Demenzkranke auch in Pflegeeinrichtungen weit höher als für Heimbewohner ohne Demenz.

\section{Dreifach erhöhtes Risiko bei vaskulärer Demenz}

Ein rund dreifach erhöhtes Erkrankungsrisiko berechneten die Experten um Wang für Patienten mit vaskulärer Demenz, für andere Demenzformen kamen sie auf eine Risikoerhöhung von $60-160 \%$. Afroamerikaner mit Demenz erkranken den Resultaten zufolge rund dreifach häufiger an COVID-19 als weiße US-Bürger, ähnliche Unterschiede zwischen den Ethnien gibt es jedoch auch bei US-Bürgern ohne Demenz.

Rund ein Viertel aller COVID-19Patienten ohne Demenz musste zwischen Februar und August 2020 stationär aufgenommen werden, mit $60 \%$ war der Anteil bei den Demenzkranken deutlich höher; von den Demenzkranken ohne COVID-19 kamen lediglich $12 \%$ in eine Klinik.

Von allen COVID-19-Patienten starben zwischen Februar und August 5,6\%. Unter den betroffenen Demenzkranken erreichte die Mortalität $21,0 \%$, zudem starben auch 7,6\% der Demenzkranken ohne COVID-19.

\section{Löchrige Bluthirnschranke}

Für das erhöhte Erkrankungsrisiko führen die Forscher um Wang einige Erklärungen ins Feld: Demenzkranke brauchen meist mehr Pflege als andere ältere Menschen und kommen dadurch häufiger in engen Kontakt mit Pflegekräften, zudem ist es für sie schwerer, 


\section{Das Wichtigste in Kürze}

Frage: Erhöht eine Demenz das Risiko, an COVID-19 zu erkranken? Antwort: Eine Demenz geht US-Daten zufolge mit einem verdoppelten Erkrankungsrisiko einher, unabhängig von Faktoren wie dem Aufenthalt in einem Pflegeheim. Bedeutung: Demenzkranke benötigen besondere Schutzmaßnahmen. Einschränkung: Ausgewertete Daten möglicherweise nicht repräsentativ.

Masken zu tragen, sich die Hände zu waschen und zu desinfizieren oder anderen Menschen aus dem Weg zu gehen.

Doch auch biologische Veränderungen könnten das Erkrankungsrisiko steigern: Eine gestörte Bluthirnschranke ermöglicht es SARS-CoV-2 vielleicht, leichter ins Gehirn zu gelangen und schwere Verläufe zu verursachen. Diese könnte vor allem auch Patienten mit vaskulärer Demenz betreffen, die den Resultaten zufolge ein besonders hohes COVID-19-Risiko haben. Bei ihnen kommt hinzu, dass viele Risikofaktoren für einen schweren Verlauf wie Diabetes, KHK, Hypertonie oder Atherosklerose vorliegen.

Genau das dürfte jedoch die Interpretation der Resultate erschweren: Die insgesamt recht hohe Mortalität von über $5 \%$ bei allen Patienten mit COVID-19-Diagnosen deutet darauf hin, dass in den elektronischen Akten vor allem schwer symptomatische COVID-19 Patienten erfasst wurden. Möglicherweise ist also nicht das Erkrankungsrisiko per se bei einer Demenz erhöht, sondern die Gefahr für einen ernsten Verlauf. Da nur bei einem winzigen Bruchteil der COVID-19-Kranken ein entsprechender Vermerk in den Akten auftauchte, ist es auch fraglich, ob es sich hier um eine repräsentative Auswahl handelt oder andere Faktoren die Resultate verzerrt haben.

\section{Literatur \\ 1. Wang QQ et al. (2021) COVID-19 and dementia: Analyses of risk, disparity, and outcomes from electronic health records in the US. Alzheimer's Dement 2021. https:// doi.org/10.1002/alz.12296}

Hinweis des Verlags. Der Verlag bleibt in Hinblick auf geografische Zuordnungen und Gebietsbezeichnungen in veröffentlichten Karten und Institutsadressen neutral.

psychopraxis.neuropraxis2021·24:147-148 https://doi.org/10.1007/s00739021-00731-2

C Springer-Verlag GmbH Austria, ein Teil von Springer Nature 2021

\section{Suizidwunsch steigt nach $\mathrm{MCl}$ - und Demenz- diagnose}

Männer mit einer frisch diagnostizierten Demenz oder einer Demenzvorstufe versuchen vermehrt, sich selbst zu töten: Die Rate von Suizidversuchen ist um bis zu $70 \%$ erhöht. Nach einiger Zeit normalisiert sich das Suizidrisiko jedoch wieder, legen US-Daten nahe.

\begin{abstract}
Sowohl die Diagnose einer beginnenden Demenz als auch die mit der Erkrankung und Prognose verbundenen Probleme können die Betroffenen erheblich erschüttern - mitunter so sehr, dass sie sich selbst töten wollen. Auf der anderen Seite sind eine Demenz und leichte kognitive Einschränkungen $(\mathrm{MCl})$ oft mit psychischen Störungen wie Depressionen verbunden, die ebenfalls das Suizidrisiko erhöhen.

Mitunter sind psychische Störungen und nicht eine Neurodegeneration der
\end{abstract}

Grund für kognitive Einschränkungen bei älteren Menschen. Nach Daten der US-Veteranenversicherung steigt die Rate von Suizidversuchen bei ehemaligen Militärangehörigen jedoch unabhängig von psychischen Diagnosen, wenn bei ihnen eine $\mathrm{MCl}$ oder Demenz diagnostiziert wird.

\section{Daten von 150.000 Veteranen}

Für die Analyse haben klinische Psychologen um Dr. Mia Maria Günak von der Universität Leiden in den Niederlanden Angaben zu rund 21.000 US-Veteranen ausgewertet, die zwischen 2011 und 2013 älter als 50 Jahre alt waren und eine MCI-Diagnose hatten [1]. Etwa die Hälfte von ihnen bekam die Diagnose in diesen beiden Jahren, sie wurden als frisch diagnostiziert bezeichnet, die übrigen hatten sie zuvor erhalten. Den MCI-Patienten gegenüber stellten sie jeweils die dreifache Zahl von Veteranen mit einer Demenz und ebenso viele ohne kognitive Probleme (Kontrollgruppe), wobei sie auf dasselbe Geschlecht, dieselbe Ethnie, ein ähnliches Alter, Einkommen und Bildungs- 